Trauma Berufskrankh 2017 · [Suppl 1]: 19:S1-S6 DOI 10.1007/s10039-016-0197-7

Online publiziert: 26. August 2016

(c) Springer-Verlag Berlin Heidelberg 2016

CrossMark
Sebastian Fischer - Berthold Bickert · Christian Andreas Radu - Benjamin Ziegler · Thomas Kremer · Ulrich Kneser · Christoph Hirche

Klinik für Hand-, Plastische und Rekonstruktive Chirurgie, Schwerbrandverletztenzentrum, Klinik für Plastische Chirurgie an der Ruprecht-Karls-Universität Heidelberg, BG Klinik Ludwigshafen, Ludwigshafen, Deutschland

\section{Aktuelle Therapie der Skaphoidfraktur}

verhältnis Männer zu Frauen 6:1 beträgt [7]. Die Skaphoidfraktur ist meistens eine Einzelverletzung, während die Kombination mit komplexen Handgelenk- und Handwurzelverletzungen in ca. $6 \%$ und das zeitgleiche Auftreten von Radiusfrakturen in ca. $4 \%$ der Fälle beobachtet werden [7].

Das Skaphoid ist zu zwei Drittel mit Knorpel überzogen und an fast allen Bewegungen des Handgelenkes beteiligt. Biomechanisch werden ca. $55 \%$ der axialen Kräfte des Handgelenkes über die radioskaphoidale Achse übertragen [8]. Selbst bei rechtzeitiger Diagnostik und Therapie ist je nach Lokalisation der Fraktur die bedeutsamste Komplikation die Pseudarthrose mit einer Rate von 10-13\%, wobei auch einzelne Fallserien mit $48 \%$ berichtet wurden $[9,10]$. Frakturen im proximalen Drittel des Skaphoids haben ein erhöhtes Risiko für die Entwicklung einer Pseudarthrose [10]. Dies ist durch die von distal in den Knochen eintretende Blutversorgung erklärt, durch welche die Perfusion nach proximal abnimmt und nach Fraktur insuffizient werden kann. Die Pseudarthrose kann bei fehlender Diagnostik und Therapie zur karpalen Instabilität und diese wiederum zum karpalen Kollaps führen (sog. „scaphoid non-union advanced collapse" [SNAC]). Trauma und pathologische Gelenkabnutzung bedingen eine Arthrose an pathognomonischen Stellen, woraus Schmerzen mit entsprechenden Kraft- und Bewegungseinschränkungen resultieren. Daten der AOK aus dem Jahr 2008 zeigen, dass für die Diagnosegruppe S62 allein im verunfallten AOK-Patientengut 19.579 Arbeitsunfähigkeitsfälle und
621.269 Arbeitsunfähigkeitstage angefallen sind. Zwar gehen - statistisch gesehen - nur $10 \%$ dieser Diagnosen auf Skaphoidfrakturen zurück, jedoch ist die Dauer der Ruhigstellung und des Arbeitsausfalles bei Skaphoidfrakturen im Vergleich zu anderen Frakturen an der Hand deutlich länger [1].

\section{Klinik}

Radiokarpale Schmerzen, Schwellung und eine hämatombedingte Verfärbung nach adäquatem Trauma des Handgelenkes können klinische Hinweise auf eine Skaphoidfraktur sein. Anhand der aktuellen Literatur sind diese Anzeichen jedoch weder ausreichend spezifisch noch sensitiv für ihre Diagnose [11]. Klinische Tests wie der Daumenstauchungsschmerz, Schmerzen bei forcierter Radialabduktion oder Supination im Handgelenk oder ein

\footnotetext{
Infobox 1 Häufigste Differenzialdiagnosen bei radiokarpalen Handgelenkschmerzen nach Trauma. (Aus $[9,11]$ )

- Frakturen:

- Intraartikulär des distalen Radius

- Skaphoid

- Styloid

- Trapezium

- Weichteile:

- Skapholunäres Band

- Arthrose:

- Radioskaphoidales Gelenk

- Skaphotrapeziotrapezoidales Gelenk

- Daumensattelgelenk
} aktive Männer zwischen dem 20. und 40. Lebensjahr, wobei das Geschlechter- 

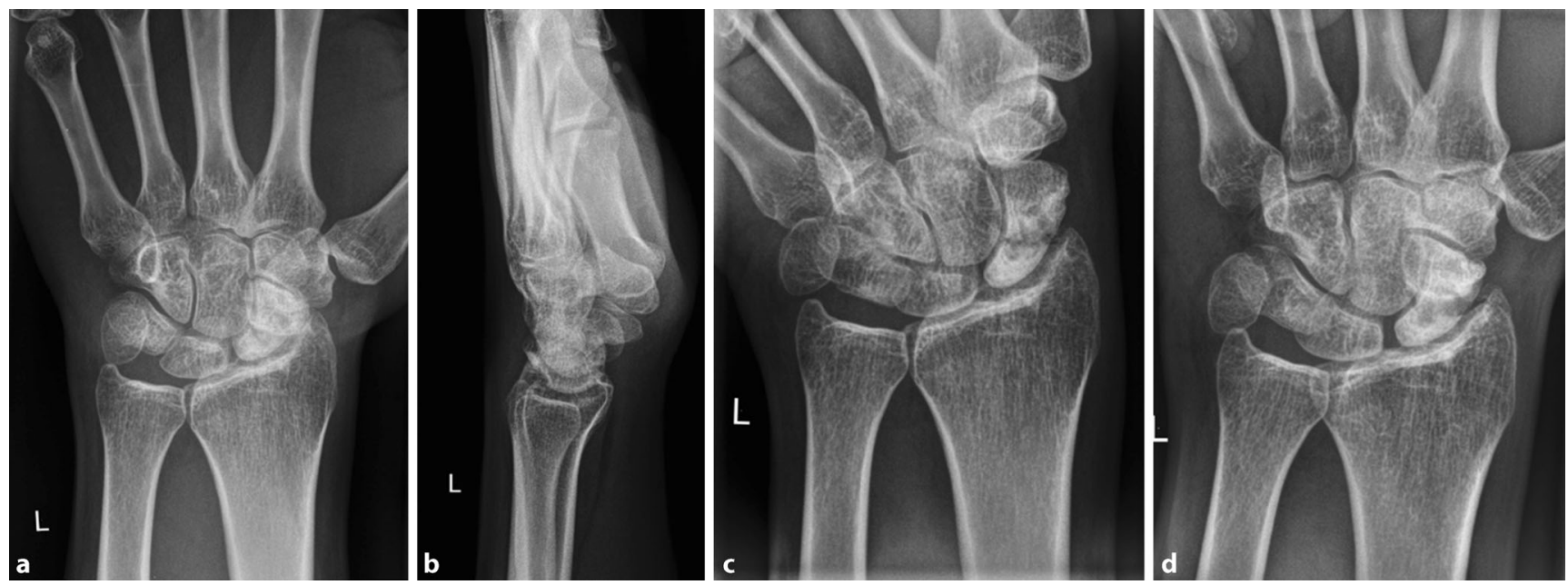

Abb. 1 ॥ Skaphoidquartett. a Posterior-anterior (p. a.), b streng seitlich, c Stecher- und d Moneim-Aufnahme
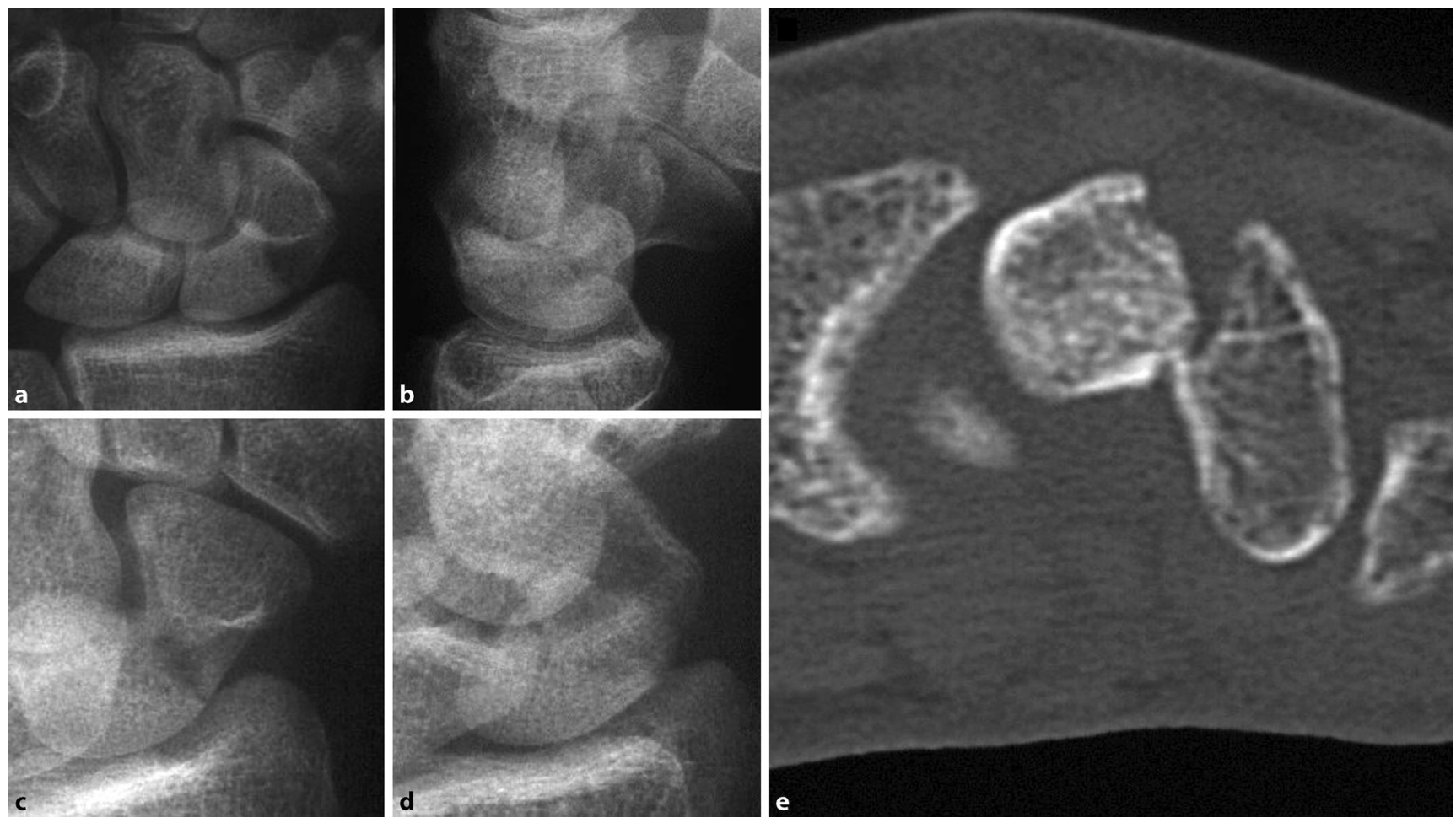

Abb. 2 ॥ Skaphoidfraktur im konventionellen Röntgen. a Posterior-anterior (p.a.), b streng seitlich, c Stecher-, d MoneimAufnahme und e in der Computertomographie (CT): Die für die Operationsplanung relevante Humpback-Deformität wird erst im CT deutlich erkenntlich

Tuberkulumdruckschmerz sind ebenfalls als gering spezifisch und wenig sensitiv einzustufen. Lediglich der Tabatiere-Druckschmerz ist als einziges klinisches Zeichen aufgrund seiner hohen Sensitivität trotz niedriger Spezifität von relevantem Nutzen [12]. Carpenter et al. [11] konnten in einer Metaanalyse zeigen, dass bei Patienten, die nach einem Sturz auf die dorsal extendierte
Hand eine Notaufnahme aufsuchen, ein Druckschmerz der Tabatiere bei radialseitigem Ruheschmerz im Handgelenk in $33 \%$ der Fälle auf eine Skaphoidfraktur hinweist. Besteht zusätzlich ein Daumenstauchungsschmerz erhöht sich die Diagnosesicherheit auf $50 \%$. Interessanterweise hatten Schwellung und hämatombedingte Verfärbungen keinen Einfluss auf die Sicherheit der Diagno- sestellung. Differenzialdiagnosen bei gegebener klinischer Symptomatik sind Frakturen der benachbarten karpalen Knochen, die skapholunäre Bandruptur sowie Aktivierung von Arthrosen (•Infobox 1). 


\section{Bildgebende Diagnostik}

Der Standard der bildgebenden, karpalen Diagnostik umfasst in erster Linie Röntgenaufnahmen in 2 Ebenen (p.-a. und streng seitlich) und zusätzliche Spezialprojektionen. Unter den Letztgenannten sollte in jedem Fall die Aufnahme in Ulnarabduktion und $20^{\circ}$ Anhebung radial (sog. Stecher-Projektion) ergänzt werden, um das Skaphoid in seiner Längsachse darzustellen. Die Aufnahme mit $20^{\circ}$ ulnarer Anhebung (sog. Moneim-Projektion) kann weiterführende Hinweise zur Beurteilung des skapholunären (SL) Spaltes liefern (• Abb. 1). Ergibt sich hier der (unsichere) Hinweis für eine Skaphoidfraktur, sollte eine Computertomographie (CT)-Diagnostik zur Beurteilung der detaillierten Frakturmorphologie erfolgen, in der das eigentliche Ausmaß einschließlich Abkippung („Humpback“) erst ersichtlich werden kann (• Abb. 2). Zeigen sich im konventionellen Röntgen keine Auffälligkeiten, sind eine Gipsruhigstellung und eine klinische sowie radiologische Reevaluation (ggf. direkt mittels CTBildgebung) innerhalb von wenigen Tagen vertretbar. Bis zu $27 \%$ der Skaphoidfrakturen sind in der initialen Röntgenbildgebung nicht detektierbar [13].

Zur optimalen Beurteilung sollte die Rekonstruktion der sagittalen Schichten im CT in der Längsachse des Skaphoids mit einer Schichtdicke von maximal $1 \mathrm{~mm}$ erfolgen. Dabei befindet sich die Hand des Patienten über seinem Kopf und kann mit geringer Strahlenbelastung untersucht werden. Die CT-Diagnostik erlaubt die hochsensitive und hochspezifische Diagnosestellung und Klassifikation der Fraktur und ist unabdingbar, um das geeignete Therapieverfahren $\mathrm{zu}$ wählen.

Der Magnetresonanztomographie (MRT) kommt in der Diagnostik der frischen Skaphoidfraktur momentan nur eine untergeordnete Rolle zu. Auch wenn sie eine hohe Sensitivität und Spezifität aufweist, so kommt sie nur bei speziellen Fragestellungen zum Einsatz [14]. Insbesondere zum Ausschluss einer Fraktur oder eines „bone bruise“ bei unklarem Röntgen- und CT-Befund trotz persis-

Trauma Berufskrankh 2017 · [Suppl 1]: 19:S1-S6 DOI 10.1007/s10039-016-0197-7

(c) Springer-Verlag Berlin Heidelberg 2016

S. Fischer - B. Bickert · C. A. Radu • B. Ziegler - T. Kremer - U. Kneser · C. Hirche

Aktuelle Therapie der Skaphoidfraktur

\section{Zusammenfassung}

Hintergrund. Die Skaphoidfraktur ist die häufigste karpale Fraktur. Betroffen sind v. a. junge Männer mit Ausfall als Arbeitskraft und Behandlung zulasten der Kranken- oder Unfallversicherungen. Die bedeutsamste Komplikation ist die Ausbildung einer Pseudarthrose bis hin zum karpalen Kollaps, die massive Einschränkungen der Handgelenkfunktion zur Folge haben kann und häufig ihre Ursachen in fehlerhafter Diagnosestellung oder unzureichender Therapie hat.

Fragestellung. Dieser Übersichts-Artikel fasst relevante Aspekte der konservativen und operativen Therapie der Skaphoidfraktur unter Berücksichtigung der wissenschaftlichen Literatur und der S3-Leitlinie zusammen. Material und Methoden. Es erfolgen eine Auswertung der amtlichen Statistiken, der relevanten und außergewöhnlichen Fälle aus dem Patientengut der Autoren, die Diskussion von aktuellen Studien und Metaanalysen sowie der S3-Leitlinie.

Ergebnisse. Der Tabatiere-Druckschmerz ist das sensitivste Zeichen in der klinischen Un- tersuchung. Die Computertomographie mit Rekonstruktion von Skaphoidlängsschichten in 0,5-1 mm Schichtdicke ist notwendig für die vollständige Diagnostik und Therapieplanung. Der Oberarmgips bietet verglichen mit der Ruhigstellung mit Handgelenkeinschluss keinen Vorteil. Die operative Therapie ist der konservativen Therapie kurz- bis mittelfristig bis 12 Monate nach Trauma bei geringerer Komplikationsrate und kürzerer Ruhigstellung überlegen. Langfristig weist sie keinen Vorteil auf.

Schlussfolgerung. Eine adäquate Versorgung ist vor dem Hintergrund der Heterogenität der Frakturformen mit jeweils unterschiedlichen Therapieempfehlungen herausfordernd und von äußerster Wichtigkeit. Aktuelle Metaanalysen und Studien erbringen neue und relevante Erkenntnisse, die in der Versorgung berücksichtigt werden sollten.

\section{Schlüsselwörter}

Handwurzelfraktur · Tabatiere-Druckschmerz . Computertomographie - Ruhigstellung . Pseudarthrose

\section{Current treatment of scaphoid bone fractures}

\section{Abstract}

Background. Scaphoid bone fractures are the most common carpal bone fractures. Young adult males are predominantly affected by scaphoid fractures leading to a high financial burden for occupational and social insurances. Scaphoid non-union up to carpal collapse is the most significant complication of untreated or undiagnosed scaphoid fractures, which can dramatically impair wrist function.

Objective. This review article summarizes the relevant aspects of conservative and surgical treatment of scaphoid bone fractures and provides an overview of current diagnostic and therapeutic recommendations and guidelines.

Material and methods. The official statistics as well as relevant and remarkable cases from the experience of the authors are analyzed.

Current studies, meta-analyses and guidelines are discussed.

Results. Tenderness on palpation of the snuffbox is the most sensitive clinical sign during physical examination. A computed tomography (CT) scan in the longitudinal axis of the scaphoid bone and a layer thickness of 0.5-1 $\mathrm{mm}$ are necessary for the diagnostics and treatment planning. An upper arm cast does not provide any advantages compared to wrist immobilization alone. Surgical treatment of non-displaced fractures is superior to conservative treatment in the short and mid-term up to 12 months after trauma due to less complications and faster mobilization; however, in the long term surgery shows no significant benefits. Conclusion. An adequate treatment of scaphoid bone fractures is important but challenging regarding the heterogeneity and variety of therapeutic options. Current meta-analyses and studies result in new and relevant information, which should be incorporated in the treatment of scaphoid fractures.

\section{Keywords}

Carpal fractures - Snuffbox tenderness . Computed tomography - Immobilization . Scaphoid non-union 


\section{Übersichten}
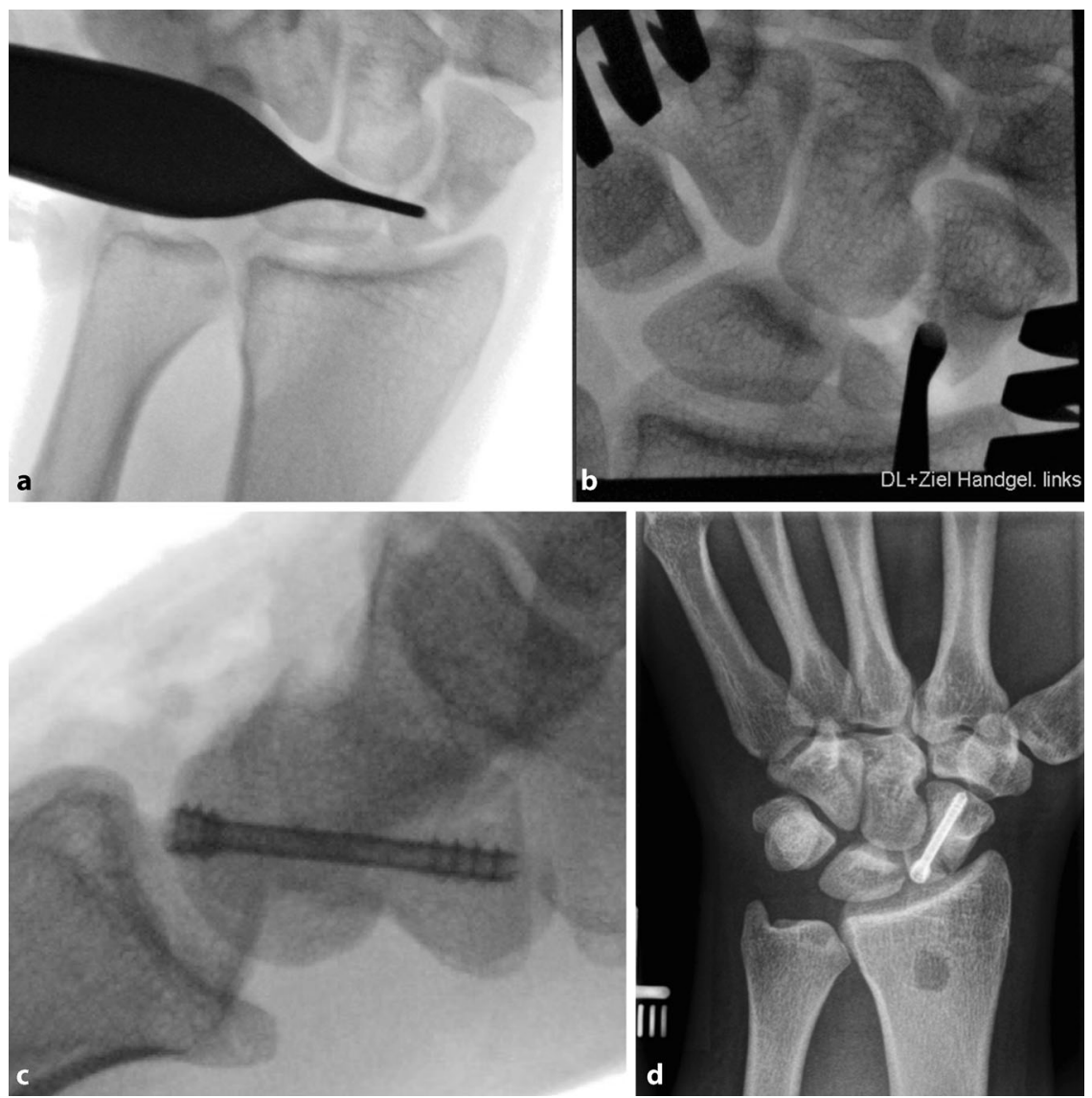

Abb. 3 A Fall einer Compactainsel im Bereich einer Skaphoidfraktur, der die Resektion und primäre Versorgung mit autologer Knochentransplantation bei der akuten Versorgung notwendig macht. a Präoperatives p.-a. Röntgenbild, b intraoperative Darstellung der Compactainsel, c intraoperative Kontrolle der Schraubenlage, d postoperatives Röntgenbild mit einliegender Herbert-Schraube und Entnahme der Radiusspongiosa

tierender Beschwerden kann das MRT hilfreich sein. Während eine unauffällige MRT eine Fraktur mit hinreichender Sicherheit ausschließt, sind (in der Regel hyperintense) Signalveränderungen $\mathrm{zu}$ unspezifisch bezüglich der detaillierten Frakturlokalisation und -konfiguration. Eine CT-Diagnostik ist somit sowohl zur Entscheidung konservative vs. operative Therapie als auch zur exakten Planung eines evtl. operativen Therapieverfahrens indiziert.

\section{Therapie}

Die Wahl der Therapie richtet sich nach Dislokationsgrad, Stabilität, Lokalisation und Morphologie der Fraktur. Die Einteilung nach Herbert/Krimmer, basierend auf dem CT-Befund, ist hierbei wegweisend [15]. Unterschieden werden stabile (Typ A) und instabile (Typ B) Frakturen, die entsprechend konservativ (Typ A) bzw. operativ (Typ B) versorgt werden sollten (• Tab. 1).

\section{Konservative Therapie}

Die Grundlage der konservativen Therapie ist die Ruhigstellung mittels Gipsoder Kunststoffschienen. Bei klinischem Verdacht auf eine Skaphoidfraktur, für die es jedoch initial radiologisch kein Korrelat gibt, ist die Gipsruhigstellung bis zur Reevaluation innerhalb 1 Woche empfehlenswert. Bei radiologisch gesicherten Skaphoidfrakturen ist prinzipiell ein konservatives Verfahren nur im Falle einer nicht dislozierten Fraktur in den distalen zwei Dritteln (A1 oder A2) oder bei kompletten Trümmerfrakturen der Pole vertretbar, wenn eine operative Reposition und interne Fixierung nicht möglich ist. Die Ruhigstellung beträgt dann in der Regel 4 (A1), 6 bis 8 (A2) bzw. 8 bis 12 Wochen mit radiologischen Kontrollen zum Ausschluss einer sekundären Dislokation alle 3 Wochen.

Der lange empfohlene Oberarmgips zeigt hierbei im Vergleich zum Unterarmgips keinen Vorteil [16] und viele Nachteile.

Bei Frakturen im proximalen Drittel besteht unter konservativer Therapie ein Risiko von 34 \% für die Entwicklung einer Pseudarthrose [10].

Shen et al. [17] verglichen in einer Metaanalyse die konservative gegen die operative Therapie der nicht oder nur minimal dislozierten Skaphoidfraktur. Innerhalb der ersten 2 bis 3 Monate zeigten sich günstigere funktionelle Ergebnisse nach operativer Therapie, während nach 6 bis 12 Monaten kein signifikanter Vorteil mehr bestand. Das Bewegungsausmaß im Handgelenk zeigte zu keinem Zeitpunkt einen signifikanten Unterschied. Der Kraftgrad war günstiger nach operativer Therapie bis 12 Monate nach Unfall. Die nichtoperative Therapie konnte als unabhängiger Risikofaktor für eine verzögerte Konsolidierung nach nichtoperativer Therapie identifiziert werden. Das Gesamtergebnis ergab jedoch keine signifikanten Unterschiede, womit beide Verfahren mittel- bis langfristig als gleichwertig anzusehen sind. Ein entscheidender Aspekt, der in dieser Studie nicht unterschieden werden konnte, ist die Lokalisation der Fraktur und das davon abhängige Risiko der Entwicklung einer Pseudarthrose. Eastley et al. [10] zeigten in einer Metaanalyse, dass die konservative Versorgung von Frakturen des proximalen Pols in $34 \%$ der Fälle in einer Pseudarthrose resultiert. Dies ist 7,5-fach häufiger im Vergleich zu Frakturen der distalen Skaphoidanteile.

\section{Operative Therapie}

Die operative Therapie mittels Reposition und interner Fixierung ist bei allen dislozierten Skaphoidfrakturen das Verfahren der Wahl [18]. Bei nicht dislozierten Frakturen ermöglicht die operative Fixierung mit limitiert offenem Zugang eine frühzeitige Freigabe zur Übungstherapie und zeigt innerhalb der ersten 3 bis 12 Monate mit hohem Evidenzlevel 
Tab. 1 Klassifikation der frischen Skaphoidfraktur nach Herbert/Krimmer. (Aus $[4,13,15])$

\begin{tabular}{|ll}
\hline \multicolumn{2}{|l}{ Typ A-Stabile Frakturen } \\
\hline A1 & Tuberkelfrakturen \\
\hline A2 & $\begin{array}{l}\text { Nicht dislozierte Rissfrakturen mit } \\
\text { querem Verlauf im mittleren oder } \\
\text { distalen Drittel }\end{array}$ \\
\hline Typ B & Instabile Frakturen \\
\hline B1 & Schrägfrakturen \\
\hline B2 & Dislozierte oder klaffende Frakturen \\
\hline B3 & Frakturen des proximalen Drittels \\
\hline B4 & $\begin{array}{l}\text { Transskaphoidale perilunäre Luxati- } \\
\text { onsfraktur }\end{array}$ \\
\hline B5 & Mehrfragmentäre Frakturen \\
\hline
\end{tabular}

aus einer Metaanalyse mit randomisiert kontrollierten Studien einen funktionellen Vorteil und eine schnellere knöcherne Konsolidierung. Dennoch gleicht sich das Behandlungsergebnis nach 12 Monaten an, sodass zu diesem Zeitpunkt keine Überlegenheit nachweisbar ist [17].

Die kanülierte, idealerweise selbstbohrende Doppelgewindekompressionsschraube in einer Modifikation nach Herbert ist hierbei das präferierte Implantat [16]. Sie erlaubt ein minimalinvasives bzw. limitiert offenes Vorgehen mit vernachlässigbarem Weichteilschaden. Die Entscheidung, ob offen oder minimalinvasiv vorgegangen werden soll, hängt vom Ausmaß der Dislokation, einer notwendigen Aufrichtung und der in seltenen Fällen auch bei der Fraktur notwendigen Transplantation von autologem Knochenmaterial ab, z. B. bei großer Zyste oder Compactainsel (• Abb. 3). Bei geschlossen nicht zu reponierender Dislokation oder Lysezonen mit der Notwendigkeit einer Knochentransplantation kommt ein offenes Verfahren in Betracht. Bei proximalem Frakturfragment wird ein dorsaler Zugang für die Schraubenpositionierung empfohlen, während im mittleren Drittel je nach Verlauf und Abkippung ein dorsaler oder palmarer Zugang gewählt werden kann. Bei den Frakturen des distalen Drittels ist ein Zugang von palmar zu empfehlen.

Für proximale Frakturen sind bei kleineren Fragmenten Doppelgewindeschrauben von kleinem Durchmesser empfehlenswert. In besonderen Fällen ist das Einbringen einer kanülierten Doppelgewindeschraube nicht möglich. Bei zur Skaphoidachse längs verlaufenden Frakturen oder Frakturen, die eine palmare Abstützung benötigen, sind winkelstabile Skaphoidplatten mit 1,5mm-Schrauben eine Rückzugsoption für die interne Fixierung, wobei dieses Implantat im Gegensatz zur Schraube in der Regel wieder entfernt werden muss.

Die Vorteile einer operativen Therapie sind die vorzeitige Wiederaufnahme der Arbeit, Bewegung im Handgelenk und Verzicht auf Ruhigstellung bei nicht dislozierten Frakturen sowie höhere Durchbauungsraten bei dislozierten Frakturen.

\section{Transskaphoidale perilunäre Luxationsfraktur (De-Quervain)}

Eine Besonderheit bietet die transskaphoidale perilunäre Luxationsfraktur (De-Quervain), die immer operativ versorgt werden muss. Diese seltene Form stellt eine komplexe Carpusverletzung dar und hat ihren Verletzungsmechanismus in der Regel in einer Hyperextension mit ulnarer Deviation und interkarpaler Supination. Es ist davon auszugehen, dass das komplette Ausmaß der Verletzung in ca. $25 \%$ aller Fälle nicht vollständig detektiert wird. Zwei Drittel aller perilunären Luxationsverletzungen gehen mit einer transossären Begleitverletzung einher, sodass das Standardverfahren die Reposition, Fixierung und ligamentäre Reparatur ist. Häufig sind ein dorsaler und palmarer Zugang notwendig einschließlich Spaltung des Karpaltunnels. Ein offenes Vorgehen ist bei dieser Verletzung überlegen [19]. Es empfiehlt sich, zunächst die Luxation zu reponieren und dann das Skaphoid intern zu fixieren. Trotz Versorgung muss in $50 \%$ der Fälle mit vorzeitiger posttraumatischer Arthrose gerechnet werden.

\section{Nachbehandlung}

In Abhängigkeit vom gewählten Therapieverfahren variiert die empfohlene Zeit der Ruhigstellung. Während konservativ behandelte Frakturen des Tuberkulums (A1) in der Regel nicht länger als 4 Wochen ruhiggestellt werden müssen, sollten unverschobene Querfrakturen (A2)

bei konservativer Therapie 6 bis 8 Wochen ruhiggestellt werden.

In der Regel wird ein Unterarmgips mit an das Daumengrundgelenk heranreichender Umfassung des 1. Mittelhandknochens gewählt [20]. Bei initial grob dislozierten Frakturen oder Frakturen mit primärer, autologer Knochentransplantation sollte postoperativ eine 6- bis 8-wöchige Ruhigstellung erfolgen.

Gering dislozierte Frakturen nach interner Fixierung sollten für maximal 2 bis 3 Wochen immobilisiert werden. Gleiches gilt für nicht dislozierte Frakturen des proximalen Pols nach operativer Therapie durch biomechanisch stabile Schraubenosteosynthese. Die operative Versorgung von nicht dislozierten Frakturen im mittleren Drittel zeigt ihre Stärke in der Nachbehandlung. Eine Ruhigstellung muss in diesen Fällen nur kurzzeitig erfolgen und hat vor diesem Hintergrund eher einen „psychologischen Effekt“. Ein Belastungsaufbau sollte jedoch auch bei dieser Form der Nachbehandlung erst nach Ablauf von 6 Wochen begonnen werden. Die funktionelle Physiotherapie kommt hier direkt und bei allen anderen Verfahren nach Abschluss der Ruhigstellung und Röntgenkontrolle zum Einsatz. Bei jeder Skaphoidfraktur sollten extreme Belastungen am Handgelenk für mindestens 12 Wochen vermieden werden.

Bei verzögerter Frakturheilung mit ausbleibender Konsolidierung sollte die Ruhigstellung maximal 12 Wochen erfolgen und dann ein Verfahrenswechsel z. B. mit autologer Knochentransplantation angestrebt werden.

\section{Fazit für die Praxis}

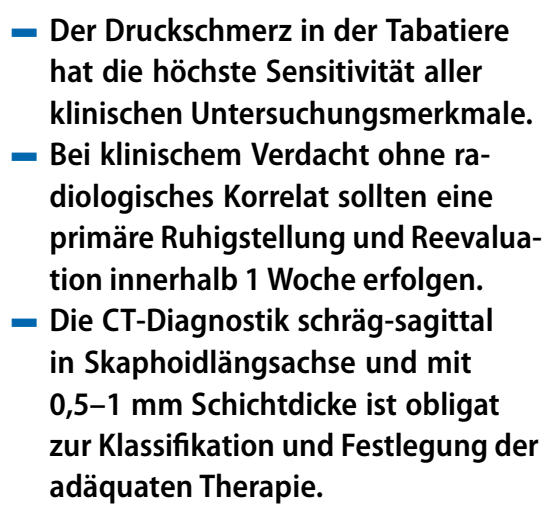




\section{- Die limitiert offene Osteosynthese ist bei nicht dislozierten Frakturen der konservativen Gipsruhigstellung in den ersten Monaten überlegen. \\ - Die Ruhigstellung soll in geringer \\ Extension des Handgelenkes unter \\ Einschluss des Metakarpale 1 in \\ lockerer Oppositionsstellung des \\ Daumenstrahls erfolgen.}

\section{Korrespondenzadresse}

\section{PD Dr. med. C. Hirche}

Klinik für Hand-, Plastische und Rekonstruktive

Chirurgie, Schwerbrandverletztenzentrum,

Klinik für Plastische Chirurgie an der Ruprecht-

Karls-Universität Heidelberg, BG Klinik

Ludwigshafen

Ludwig-Guttmann-Str. 13, 67071 Ludwigs-

hafen, Deutschland

christoph.hirche@bgu-ludwigshafen.de

\section{Einhaltung ethischer Richtlinien}

Interessenkonflikt. S. Fischer, B. Bickert, C.A. Radu, B. Ziegler, T. Kremer, U. Kneser und C. Hirche geben an, dass kein Interessenkonflikt besteht.

Dieser Beitrag beinhaltet keine von den Autoren durchgeführten Studien an Menschen oder Tieren.

The supplement containing this article is not sponsored by industry.

\section{Literatur}

1. Hove LM (1999) Epidemiology of scaphoid fractures in Bergen, Norway. Scand J Plast Reconstr Surg Hand Surg 33:423-426

2. DGUV (2012) Anfrage 1. Febr. 2012

3. Statistisches Bundesamt (2012) Anfrage 1. Febr. 2012

4. Weber ER, Chao EY (1978) An experimental approach to the mechanism of scaphoid waist fractures.J Hand Surg Am 3:142-148

5. Rongieres M, Mansat M, Bonnevialle P et al (1988) Pathomechanics of fractures of the scaphoid. Rev Chir Orthop Reparatrice Appar Mot 74:689-692

6. Duckworth $A D$, Jenkins PJ, Aitken $S A$ et al (2012) Scaphoid Fracture Epidemiology. J Trauma 72(2):E41-E45

7. Towfigh H (2011) Handchirurgie. Springer, Heidelberg

8. Schuind F, Cooney WP, Linscheid RL et al (1995) Force and pressure transmission through the normal wrist. A theoretical two-dimensional study in the posteroanterior plane. J Biomech 28:587-601

9. Barton NJ (1992) Twenty questions about scaphoid fractures.J Hand Surg Br 17:289-310

10. Eastley N, Singh H, Dias JJ et al (2013) Union rates after proximal scaphoid fractures; meta-analyses and review of available evidence. J Hand Surg Eur Vol 38(8):888-897
11. CarpenterCR, Pines JM, Schuur JD et al (2014) Adult scaphoid fracture. Acad Emerg Med 21:101-121

12. Mallee WH, Henny EP, van Dijk CN et al (2014) Clinical diagnostic evaluation for scaphoid fractures: a systematic review and meta-analysis. JHand Surg Am 39(9):1683-1691.e2

13. Jenkins PJ, Slade K, Huntley JS et al (2008) A comparative analysis of the accuracy, diagnostic uncertainty and cost of imaging modalities in suspected scaphoid fractures. Injury 39:768-774

14. Rhemrev SJ, Ootes D, Beeres FJ et al (2011) Current methods of diagnosis and treatment of scaphoid fractures. Int JEmerg Med 4:4

15. Krimmer H, Schmitt R, Herbert T (2000) Scaphoid fractures - diagnosis, classification and therapy. Unfallchirurg 103:812-819

16. Alshryda S, Shah A, Odak S et al (2012) Acute fractures of the scaphoid bone: Systematic review and meta-analysis. Surgeon 10:218-229

17. Shen L, Tang J, Luo C et al (2015) Comparison of operative and non-operative treatment of acute undisplaced or minimally-displaced scaphoid fractures: a meta-analysis of randomized controlled trials. PLOSONE 10:e0125247

18. Cheung JP, Tang CY, Fung BK (2013) Current management of acute scaphoid fractures: a review. Hong Kong Med J 20:52-58

19. Kremer T, Wendt $M$, Riedel $K$ et al (2010) Open Reduction for perilunate injuries - clinical outcome and patient satisfaction. J Hand Surg Am 35:1599-1606

20. AWMF, S3-Leitlinie Skaphoidfraktur, Leitlinien RegisterNr.012-016 